\title{
Visual selective attention with virtual barriers
}

\author{
Darryl W. Schneider ${ }^{1}$
}

Published online: 5 May 2017

(C) The Psychonomic Society, Inc. 2017

\begin{abstract}
Previous studies have shown that interference effects in the flanker task are reduced when physical barriers (e.g., hands) are placed around rather than below a target flanked by distractors. One explanation of this finding is the referential coding hypothesis, whereby the barriers serve as reference objects for allocating attention. In five experiments, the generality of the referential coding hypothesis was tested by investigating whether interference effects are modulated by the placement of virtual barriers (e.g., parentheses). Modulation of flanker interference was found only when target and distractors differed in size and the virtual barriers were beveled wood-grain objects. Under these conditions and those of previous studies, the author conjectures that an impression of depth was produced when the barriers were around the target, such that the target was perceived to be on a different depth plane than the distractors. Perception of depth in the stimulus display might have led to referential coding of the stimuli in three-dimensional (3-D) space, influencing the allocation of attention beyond the horizontal and vertical dimensions. This 3-D referential coding hypothesis is consistent with research on selective attention in 3-D space that shows flanker interference is reduced when target and distractors are separated in depth.
\end{abstract}

Keywords Selective attention · Flanker task · Interference . Referential coding $\cdot$ Depth cues

Darryl W. Schneider

dws@purdue.edu

1 Department of Psychological Sciences, Purdue University, 703 Third Street, West Lafayette, IN 47907, USA
Selective attention is important for visual cognition because it enables people to focus on targets and ignore distractors in the environment. However, there are limits to selective attention, as indicated when distractors interfere with target processing. An example of this interference occurs in the flanker task (Eriksen \& Eriksen, 1974), in which subjects identify a central target flanked by distractors associated with the same response as the target (compatible), an alternative response (incompatible), or no response (neutral). A common finding is an interference effect: response times (RTs) are longer on incompatible trials than on neutral or compatible trials. The interference effect is generally interpreted as evidence that subjects cannot restrict attention exclusively to the target, resulting in response competition from incompatible flankers (Gratton, Coles, Sirevaag, Eriksen, \& Donchin, 1988).

Recent research has shown that flanker interference is modulated by where subjects' hands are positioned relative to the target and distractors (Bush \& Vecera, 2016; Davoli \& Brockmole, 2012; Murchison \& Proctor, 2015, 2016; Weidler \& Abrams, 2014). For example, in Davoli and Brockmole's study, interference effects were reduced when subjects' hands were positioned around the target (such that the target was inside and the distractors were outside the space between the palms of the hands), relative to conditions in which the hands were below and away from the stimulus display. This finding was interpreted as evidence for what I call the hand space hypothesis, whereby the space between the hands serves as an attentional window that enhances target processing and protects against distractor interference. This idea is consistent with studies showing that visual attention and perception are modulated by hand placement (Abrams, Davoli, Du, Knapp, \& Paull, 2008; Tseng, Bridgeman, \& Juan, 2012).

Murchison and Proctor (2015) replicated and extended Davoli and Brockmole's (2012) study in two important 
respects. First, they found that interference effects were reduced regardless of whether the stimuli inside or outside of hand space were designated as the targets, indicating that the space between the hands was not privileged. Second, they found similar modulation of interference effects when wooden blocks were used instead of hands (cf. Davoli \& Brockmole, 2012), suggesting that physical barriers besides hands can influence selective attention. In follow-up work, Murchison and Proctor (2016) extended these results to outward-facing hands and wooden blocks. These findings challenged the hand space hypothesis and were interpreted as evidence for a referential coding hypothesis, whereby physical barriers (hands or wooden blocks) serve as reference objects for allocating visual attention to targets occurring inside or outside of the barriers. This idea is consistent with studies showing that other types of compatibility effects can be understood in terms of referential coding (e.g., Hommel, 1993).

The purpose of the present study was to test the generality of the referential coding hypothesis. If the critical feature of the physical barriers used previously was that they were reference objects for differentially allocating attention to targets and distractors, then similar modulation of flanker interference should be possible with nonphysical barriers that can also serve as reference objects. To investigate this idea, I conducted five experiments in which virtual barriers (e.g., parentheses) appeared onscreen around or below the target in a flanker task, analogous to barrier placement manipulations in previous studies.

\section{Experiments 1 and 2}

Subjects performed the letter version of the flanker task used in previous studies (Davoli \& Brockmole, 2012; Murchison \& Proctor, 2015, 2016). The task was to identify a central target letter flanked by compatible, incompatible, or neutral distractor letters. A key manipulation was the placement of virtual barriers (parentheses) either around or below the target (see Fig. 1). If the referential coding hypothesis generalizes

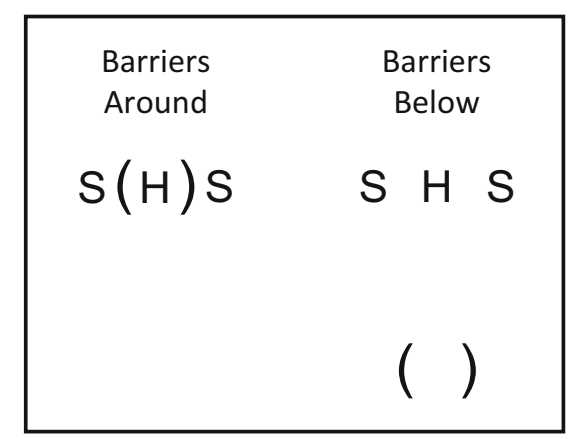

Figure 1 Examples of stimuli and barrier placement for incompatible stimulus displays in Experiments 1 and 2. Stimuli were presented in white on a black background beyond physical barriers, then one would predict smaller interference effects in the barriers-around condition than in the barriers-below condition. The only difference between experiments was whether a fixation cross appeared at the target location prior to stimulus onset, replicating conditions examined by Murchison and Proctor (2015).

\section{Method}

Subjects A total of 96 students (48 per experiment) from Purdue University participated for course credit. ${ }^{1}$ All subjects in the present study reported having normal or corrected-tonormal vision.

Apparatus The experiments were conducted on computers that displayed stimuli on a black background $\left(0.19 \mathrm{~cd} / \mathrm{m}^{2}\right)$ on 21.5-in. LED monitors and registered responses from Chronos response devices (Psychology Software Tools, Inc.). Responses involved pressing the leftmost and rightmost buttons on the response device with the left and right index fingers, respectively. Viewing distance was uncontrolled but approximately $50 \mathrm{~cm}$.

Task and stimuli The task was to identify a central target letter that was flanked on the left and the right by a distractor letter. The target letters were $H$ and $S$, which were assigned to left and right button responses, respectively (mappings were counterbalanced across subjects). The distractor letters were $H, S$, and $X$, the last of which had no assigned response. Compatibility was coded by whether the distractors were associated with the same response as the target (compatible; $H H H$ or $S S S$ ), the alternative response (incompatible; $S H S$ or $H S H$ ), or no response (neutral; $X H X$ or $X S X$ ). Letters appeared in white 18-point Arial font $\left(142.6 \mathrm{~cd} / \mathrm{m}^{2}\right)$ and were approximately $0.3 \mathrm{~cm}$ wide $\times 0.4 \mathrm{~cm}$ high; adjacent letters had centerto-center separation of $0.9 \mathrm{~cm}$. The barrier stimuli were parentheses in white 24-point Arial font; each parenthesis was $0.2 \mathrm{~cm} \times 0.7 \mathrm{~cm}$, and the outer edges of the parentheses were $1.1 \mathrm{~cm}$ apart. Barrier placement was manipulated as shown in Fig. 1. In the barriers-around condition, the parentheses were centered around the target, separating it from the distractors. In the barriers-below condition, the parentheses were $2.5 \mathrm{~cm}$ (center-to-center separation) below the target.

Procedure Subjects were tested individually after giving informed consent for a study protocol approved by the Purdue University Institutional Review Board. Instructions about the task, stimuli, and responses were presented onscreen and read

\footnotetext{
${ }^{1}$ A power analysis indicated 48 subjects would provide $98 \%$ power to detect the mean effect size of $\eta_{\mathrm{p}}{ }^{2}=.08$ for the interaction between barrier placement and compatibility found in previous experiments involving within-subjects manipulations of barrier placement (Davoli \& Brockmole, 2012; Murchison \& Proctor, 2015).
} 
aloud by the experimenter. Subjects were informed that parentheses would be displayed either around or below the central letter on each trial, but that the parentheses could be ignored. During the instructional phase, subjects did 12 practice trials (two for each possible combination of letter stimuli) with trialwise accuracy feedback but without any barriers present. During the experimental phase, they did six blocks of 48 trials per block without accuracy feedback. The first three blocks involved the barriers-around condition, and the last three blocks involved the barriers-below condition (condition order was counterbalanced across subjects). Each block consisted of all possible combinations of letter stimuli presented equally often every 24 trials in random order, which meant that all trial types (compatible, incompatible, and neutral) occurred equally often.

In Experiment 1 (fixation absent), each trial started with the simultaneous onset of all elements of the stimulus display (letters and barriers). This display remained onscreen until the subject responded, then the letters were removed (the barriers stayed visible), and the next trial commenced after a 1,000-ms response-stimulus interval. In Experiment 2 (fixation present), each trial started with a white fixation cross at the forthcoming target location for $500 \mathrm{~ms}$. The remaining trial events were the same as in Experiment 1, except the postresponse interval during which only the barriers were visible was $500 \mathrm{~ms}$, thereby maintaining a 1,000-ms responsestimulus interval. Subjects were instructed to respond quickly and accurately.

\section{Results}

Trials with RTs more than three standard deviations above the mean in each condition for a given subject were excluded (1.9\% and $1.7 \%$ of trials in Experiments 1 and 2, respectively). Error trials were excluded from the RT analyses. Mean correct RTs and error rates are summarized in Table 1. The data were submitted to 2 (barrier placement: around or below) $\times 3$ (compatibility: compatible, incompatible, or neutral) repeated-measures analyses of variance (ANOVAs), the results of which are summarized in Table 2.

Significant compatibility effects were partitioned into interference and facilitation components by using performance on neutral trials as a baseline. Interference effects were calculated as performance differences between incompatible and neutral trials (positive numbers indicate costs for incompatible trials), and facilitation effects were calculated as performance differences between compatible and neutral trials (negative numbers indicate benefits for compatible trials). Contrasts were conducted to assess interference and facilitation effects. Bayes factor analyses using scaled JZS Bayes factors with $r$ = 1 (Rouder, Speckman, Sun, Morey, \& Iverson, 2009) were conducted to quantify the evidence for or against differences in interference or facilitation effects as a function of barrier placement.

Significant main effects of compatibility on RTs were obtained in both experiments; no other effects were significant (see Table 2). In Experiment 1, the compatibility effect reflected a significant interference effect of $21 \mathrm{~ms}, t(47)=$ $7.20, p<.001, d=1.04$, and a nonsignificant facilitation effect of $1 \mathrm{~ms}, t(47)=0.21, p=.83$. As shown in the top-left panel of Fig. 2, interference effects were similar in the barriers-around and barriers-below conditions (19 $\mathrm{ms}$ and $24 \mathrm{~ms}$, respectively), and a Bayes factor analysis indicated that no difference in interference effects between conditions was 6.13 times more likely than was a difference.

In Experiment 2, the compatibility effect reflected a significant interference effect of $17 \mathrm{~ms}, t(47)=6.02, p<.001, d=$ 0.87 , and a nonsignificant facilitation effect of $-5 \mathrm{~ms}, t(47)=$ $1.72, p=.09$. As shown in the top-right panel of Fig. 2, interference effects were similar in the barriers-around and barriers-below conditions (18 $\mathrm{ms}$ and $15 \mathrm{~ms}$, respectively), and a Bayes factor analysis indicated that no difference in interference effects between conditions was 6.06 times more likely than was a difference. ${ }^{2}$

\section{Discussion}

Both experiments yielded robust compatibility effects that were mostly due to flanker interference rather than to facilitation. However, interference effects were not modulated by virtual barrier placement (see Fig. 2), regardless of whether a fixation cross appeared at the target location prior to stimulus onset. Before concluding that the referential coding hypothesis might not generalize beyond physical barriers, I report additional experiments addressing potential methodological issues.

\section{Experiments 3-5}

Flanker interference decreases as targets and flankers are spaced farther apart (Eriksen \& Eriksen, 1974; Miller, 1991). To ensure sizable interference effects would be found, stimuli were small and their spacing was narrow in Experiments 1 and 2. However, this could have constrained visual attention to such a small area that the virtual barriers were not used as reference objects for allocating attention. Note that the stimuli were larger - with the distractors larger than the targets - and spaced farther apart in previous studies to accommodate physical barriers (Davoli \& Brockmole, 2012; Murchison \& Proctor, 2015, 2016). The physical barriers were also

\footnotetext{
${ }^{2}$ Mean error rates were $3.6 \%$ and $3.1 \%$ in Experiments 1 and 2, respectively. The error data do not contradict the RT data or indicate speed-accuracy tradeoffs (see Tables 1 and 2).
} 
Table 1 Mean correct response times (in milliseconds) and error rates (as percentages)

\begin{tabular}{|c|c|c|c|c|}
\hline Experiment & Barrier placement & Compatibility & Response time & Error rate \\
\hline \multirow[t]{6}{*}{1} & \multirow[t]{3}{*}{ Around } & Incompatible & $438(11)$ & $5.3(0.6)$ \\
\hline & & Neutral & $420(10)$ & $2.9(0.5)$ \\
\hline & & Compatible & $424(11)$ & $3.0(0.6)$ \\
\hline & \multirow[t]{3}{*}{ Below } & Incompatible & $452(11)$ & $5.2(0.7)$ \\
\hline & & Neutral & $429(10)$ & $2.7(0.4)$ \\
\hline & & Compatible & $426(10)$ & $2.5(0.5)$ \\
\hline \multirow[t]{6}{*}{2} & \multirow[t]{3}{*}{ Around } & Incompatible & $447(9)$ & $4.2(0.6)$ \\
\hline & & Neutral & $428(9)$ & $2.6(0.6)$ \\
\hline & & Compatible & $424(9)$ & $2.7(0.5)$ \\
\hline & \multirow[t]{3}{*}{ Below } & Incompatible & $447(8)$ & $4.6(0.6)$ \\
\hline & & Neutral & $432(8)$ & $2.0(0.4)$ \\
\hline & & Compatible & $426(8)$ & $2.4(0.3)$ \\
\hline \multirow[t]{6}{*}{3} & \multirow[t]{3}{*}{ Around } & Incompatible & $396(9)$ & $5.2(0.8)$ \\
\hline & & Neutral & $386(10)$ & $2.6(0.4)$ \\
\hline & & Compatible & $379(8)$ & $2.8(0.5)$ \\
\hline & \multirow[t]{3}{*}{ Below } & Incompatible & $407(9)$ & $4.4(0.6)$ \\
\hline & & Neutral & $393(9)$ & $2.8(0.5)$ \\
\hline & & Compatible & $388(8)$ & $2.7(0.5)$ \\
\hline \multirow[t]{6}{*}{4} & \multirow[t]{3}{*}{ Around } & Incompatible & $391(8)$ & $5.0(0.7)$ \\
\hline & & Neutral & $386(8)$ & $3.9(0.6)$ \\
\hline & & Compatible & $376(7)$ & $3.7(0.5)$ \\
\hline & \multirow[t]{3}{*}{ Below } & Incompatible & $406(9)$ & $5.6(0.8)$ \\
\hline & & Neutral & $389(9)$ & $3.3(0.6)$ \\
\hline & & Compatible & $393(10)$ & $3.6(0.5)$ \\
\hline \multirow[t]{6}{*}{5} & \multirow[t]{3}{*}{ Around } & Incompatible & $396(12)$ & $3.3(0.5)$ \\
\hline & & Neutral & $388(10)$ & $2.8(0.5)$ \\
\hline & & Compatible & $384(8)$ & $2.5(0.4)$ \\
\hline & \multirow[t]{3}{*}{ Below } & Incompatible & 404 (13) & $4.3(0.6)$ \\
\hline & & Neutral & 393 (12) & $2.3(0.4)$ \\
\hline & & Compatible & 388 (12) & $2.5(0.4)$ \\
\hline
\end{tabular}

Note. Standard errors of the means appear in parentheses

perceptually distinct from the stimuli, in contrast with the virtual barriers being in the same white font as the letter stimuli in Experiments 1 and 2. To address these methodological differences, I replicated Experiment 1 using large stimuli spaced far apart, with (Experiments 3 and 4) or without (Experiment 5) the distractors being larger than the targets. In addition, I used more salient virtual barriers (see Fig. 3): green parentheses (Experiment 3) or beveled wood-grain objects (Experiments 4 and 5).

\section{Method}

Subjects A total of 144 students (48 per experiment) from Purdue University participated for course credit. None of them had participated in Experiments 1 or 2.
Apparatus The apparatus was identical to that used in Experiments 1 and 2.

Task and stimuli These methodological details were identical to those of Experiments 1 and 2, except letter stimuli were larger and different barrier stimuli were used. Distractor letters appeared in white 160-point Arial font and were approximately $3.0 \mathrm{~cm} \times 3.8 \mathrm{~cm}$. Target letters in Experiments 3 and 4 appeared in white 72-point Arial font and were approximately $1.3 \mathrm{~cm} \times 1.7 \mathrm{~cm}$, whereas target letters in Experiment 5 were the same size as distractor letters. Adjacent letters had centerto-center separation of $7.7 \mathrm{~cm}$. Letter widths and separation in Experiments 3 and 4 matched the measurements reported by Davoli and Brockmole (2012). The barrier stimuli are shown in Fig. 3. In Experiment 3, the barriers were parentheses in bright green 360-point Arial font $\left(103.8 \mathrm{~cd} / \mathrm{m}^{2}\right)$; each 
Table 2 Summary of analyses of variance

\begin{tabular}{|c|c|c|c|c|c|c|c|c|}
\hline \multirow[t]{2}{*}{ Experiment } & \multirow[t]{2}{*}{ Effect } & \multirow[t]{2}{*}{$d f$} & \multicolumn{3}{|c|}{ Response time } & \multicolumn{3}{|c|}{ Error rate } \\
\hline & & & $F$ & $M S E$ & $\eta_{\mathrm{p}}^{2}$ & $F$ & MSE & $\eta_{\mathrm{p}}^{2}$ \\
\hline \multirow[t]{3}{*}{1} & $\mathrm{~B}$ & 1,47 & 1.68 & 3,050 & .04 & 0.53 & 7 & .01 \\
\hline & $\mathrm{C}$ & 2,94 & $34.45^{*}$ & 399 & .42 & $19.73 *$ & 10 & .30 \\
\hline & $\mathrm{B} \times \mathrm{C}$ & 2,94 & 2.00 & 369 & .04 & 0.11 & 7 & $<.01$ \\
\hline \multirow[t]{3}{*}{2} & B & 1,47 & 0.15 & 1,666 & $<.01$ & 0.45 & 8 & .01 \\
\hline & $\mathrm{C}$ & 2,94 & $32.37 *$ & 391 & .41 & $12.55^{*}$ & 10 & .21 \\
\hline & $\mathrm{B} \times \mathrm{C}$ & 2,94 & 0.39 & 178 & .01 & 1.22 & 6 & .03 \\
\hline \multirow[t]{3}{*}{3} & $\mathrm{~B}$ & 1,47 & 2.86 & 1,787 & .06 & 0.60 & 5 & .01 \\
\hline & $\mathrm{C}$ & 2,94 & $29.66^{*}$ & 269 & .39 & $16.55^{*}$ & 8 & .26 \\
\hline & $\mathrm{B} \times \mathrm{C}$ & 2,94 & 0.21 & 405 & .01 & 0.86 & 8 & .02 \\
\hline \multirow[t]{3}{*}{4} & B & 1,47 & $5.01 *$ & 1,906 & .10 & 0.02 & 11 & $<.01$ \\
\hline & $\mathrm{C}$ & 2,94 & $22.38 *$ & 226 & .32 & $12.42 *$ & 8 & .21 \\
\hline & $\mathrm{B} \times \mathrm{C}$ & 2,94 & $3.78 *$ & 344 & .07 & 1.18 & 9 & .02 \\
\hline \multirow[t]{3}{*}{5} & B & 1,47 & 0.49 & 5,278 & .01 & 0.60 & 5 & .01 \\
\hline & $\mathrm{C}$ & 2,94 & $10.31^{*}$ & 504 & .18 & $5.71 *$ & 9 & .11 \\
\hline & $\mathrm{B} \times \mathrm{C}$ & 2,94 & 0.50 & 200 & .01 & 2.34 & 6 & .05 \\
\hline
\end{tabular}

$* p<.05$

Note. $\mathrm{B}=$ barrier placement; $\mathrm{C}=$ compatibility

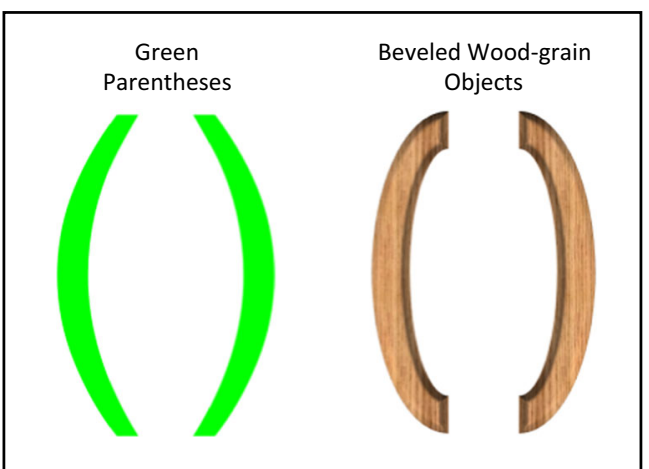

Figure 3 Barrier stimuli used in Experiments 3-5. Stimuli were presented on a black background

parenthesis was $2.8 \mathrm{~cm} \times 11.2 \mathrm{~cm}$, and the outer edges of the parentheses were $8.4 \mathrm{~cm}$ apart. In Experiments 4 and 5, the barriers were curved objects with beveled edges and a woodgrain texture (averaging $42.9 \mathrm{~cd} / \mathrm{m}^{2}$ ); they had similar dimensions but slightly different curvatures than the parentheses used in Experiment 3. To accommodate the larger barriers on the screen (which still had a black background), letter stimuli appeared in the top half of the screen. In the barriersaround condition, the barriers were centered around the target, separating it from the distractors. In the barriers-below
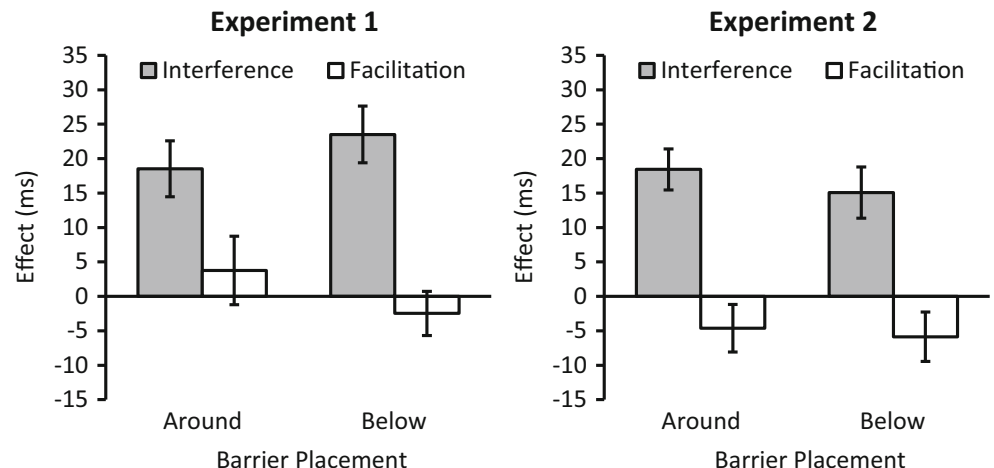

Experiment 3

Experiment 4
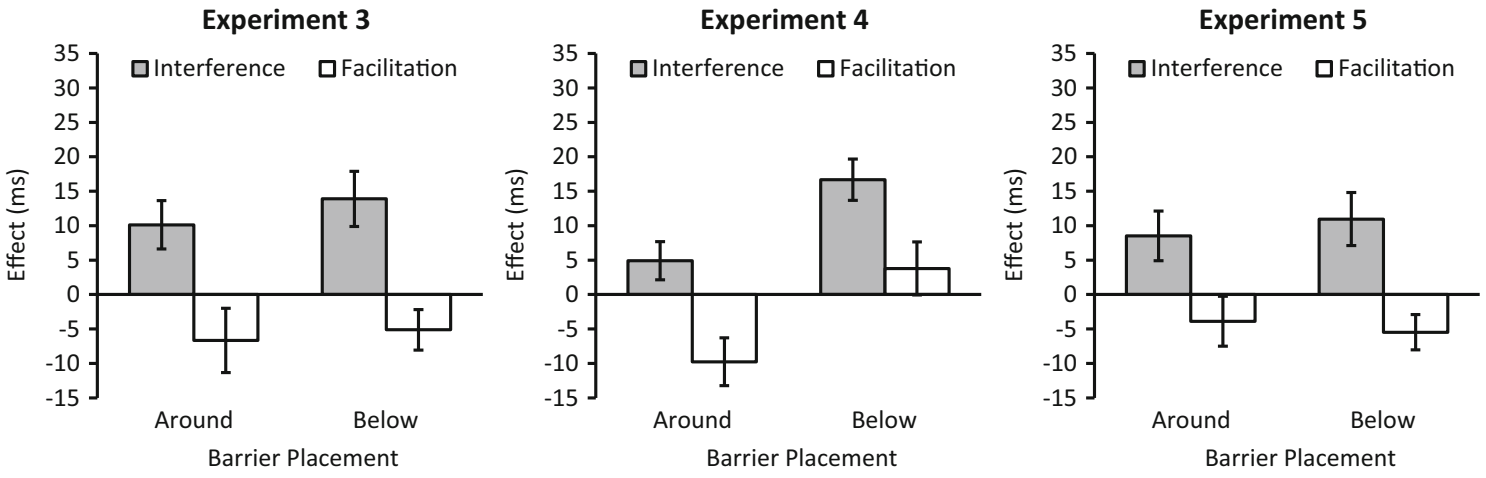

Figure 2 Mean interference and facilitation effects on response times (RTs) as a function of barrier placement. The interference effect is the difference between incompatible and neutral RTs (positive numbers indicate costs for incompatible trials), and the facilitation effect is the

difference between compatible and neutral RTs (negative numbers indicate benefits for compatible trials). Error bars represent standard errors of the means 
condition, the barriers were $12.5 \mathrm{~cm}$ (center-to-center separation) below the target but fully visible onscreen.

Procedure The procedure was identical to that of Experiment 1, except the instructions for Experiments 4 and 5 referenced "curved wooden objects" rather than parentheses.

\section{Results}

Data trimming was identical to that of Experiments 1 and 2 $(2.0 \%, 1.9 \%$, and $1.9 \%$ of trials in Experiments 3, 4, and 5, respectively, were excluded as RT outliers). Mean correct RTs and error rates are summarized in Table 1 . The data were submitted to 2 (barrier placement: around or below) $\times 3$ (compatibility: compatible, incompatible, or neutral) repeatedmeasures ANOVAs, the results of which are summarized in Table 2.

Significant main effects of compatibility on RTs were obtained in all experiments (see Table 2). In Experiment 3, the compatibility effect reflected a significant interference effect of $12 \mathrm{~ms}, t(47)=6.64, p<.001, d=0.96$, and a significant facilitation effect of $-6 \mathrm{~ms}, t(47)=2.42, p=.02, d=0.35$. As shown in the bottom-left panel of Fig. 2, effects were similar in the barriers-around and barriers-below conditions (interference effects: $10 \mathrm{~ms}$ and $14 \mathrm{~ms}$; facilitation effects: $-7 \mathrm{~ms}$ and $-5 \mathrm{~ms}$ ), and Bayes factor analyses indicated that no differences in interference and facilitation effects between conditions were respectively 7.55 and 8.58 times more likely than were differences.

In Experiment 4, the compatibility effect reflected a significant interference effect of $11 \mathrm{~ms}, t(47)=4.90, p<.001, d=$ 0.71 , and a nonsignificant facilitation effect of $-3 \mathrm{~ms}, t(47)=$ $1.39, p=.17$. The main effect of barrier placement and the interaction between barrier placement and compatibility were also significant (see Table 2). The barrier placement effect reflected shorter RTs in the barriers-around condition (385 $\mathrm{ms})$ than in the barriers-below condition $(396 \mathrm{~ms})$. The interaction is depicted in the bottom-middle panel of Fig. 2. The interference effect was smaller in the barriers-around condition $(5 \mathrm{~ms})$ than in the barriers-below condition $(17 \mathrm{~ms})$, and a Bayes factor analysis indicated that a difference in interference effects between conditions was 9.44 times more likely than was no difference. Numerically, the facilitation effect was more pronounced in the barriers-around condition $(-10 \mathrm{~ms})$ than in the barriers-below condition (4 ms), but a Bayes factor analysis indicated that a difference in facilitation effects between conditions was only 1.26 times more likely than was no difference. Thus, the interaction was driven by the difference in interference effects.

In Experiment 5, the compatibility effect reflected a significant interference effect of $10 \mathrm{~ms}, t(47)=2.87, p=.006, d=$ 0.41 , and a significant facilitation effect of $-5 \mathrm{~ms}, t(47)=2.13$, $p=.04, d=0.31$. As shown in the bottom-right panel of Fig. 2 , effects were similar in the barriers-around and barriers-below conditions (interference effects: $9 \mathrm{~ms}$ and $11 \mathrm{~ms}$; facilitation effects: $-4 \mathrm{~ms}$ and $-5 \mathrm{~ms}$ ), and Bayes factor analyses indicated that no differences in interference and facilitation effects between conditions were respectively 6.61 and 8.32 times more likely than were differences. ${ }^{3}$

\section{Discussion}

Robust compatibility effects (mostly reflecting interference) were obtained with large stimuli spaced far apart, replicating the previous experiments involving small stimuli spaced close together. Interference effects were not modulated by the placement of green parentheses for different-sized targets and distractors in Experiment 3, or by the placement of beveled wood-grain objects for equal-sized targets and distractors in Experiment 5. However, interference effects were reliably modulated by the placement of beveled wood-grain objects for different-sized targets and distractors in Experiment 4, similar to the effects of physical barriers in previous studies. A possible explanation for the present data is offered in the next section.

\section{General discussion}

I conducted five experiments to test the generality of the referential coding hypothesis for why flanker interference was modulated by physical barrier placement in previous studies (Davoli \& Brockmole, 2012; Murchison \& Proctor, 2015, 2016). Virtual barriers were placed around or below the central target in a flanker task, but modulation of interference effects occurred only in Experiment 4, which involved large stimuli-with the distractors larger than the targets - and beveled wood-grain objects as virtual barriers. These results challenge the hand space hypothesis and suggest that the referential coding hypothesis generalizes beyond physical barriers under specific conditions. In this section, I propose a variant of the referential coding hypothesis to account for the present and previous data.

A feature of the virtual barriers in Experiments 4 and 5 is that their beveled edges and wood-grain texture suggested a three-dimensional (3-D) appearance, which may have induced subjects to perceive depth in the stimulus display. To improve perception of the peripheral flankers under wider stimulus spacing, the distractors were larger than the target in

\footnotetext{
${ }^{3}$ Mean error rates were 3.4\%, 4.2\%, and 3.0\% in Experiments 3, 4, and 5, respectively. The error data do not contradict the RT data or indicate speedaccuracy trade-offs (see Tables 1 and 2). The interaction between barrier placement and compatibility was nonsignificant in the error data of Experiment 4, but the interference effect was numerically smaller in the barriers-around condition $(1.1 \%)$ than in the barriers-below condition (2.4\%), consistent with the RT data.
} 
Experiments 3 and 4 (following Davoli \& Brockmole, 2012; Murchison \& Proctor, 2015, 2016). Relative size is a wellknown pictorial depth cue, such that for two similar objects (letters in the present context), the one casting a smaller image on the retina is perceived as farther away (Palmer, 1999). I conjecture that the combination of these elements-3-D virtual barriers separating the letter stimuli and the relative size difference between target and distractor letters - in the barriers-around condition in Experiment 4 was sufficient to convey the impression that the target was on a different (more distant) depth plane than the flanking distractors. I hypothesize that perception of depth in the stimulus display might have led to referential coding of the stimuli in 3-D space, influencing the allocation of attention beyond the horizontal and vertical dimensions. This 3-D referential coding hypothesis, which can be considered a variant of the original referential coding hypothesis (Murchison \& Proctor, 2015, 2016), also applies to previous studies in which physical barriers were 3-D elements of the display context and the stimuli differed in size.

The 3-D referential coding hypothesis can explain why flanker interference is modulated by 3-D barrier placement based on previous research concerning visual selective attention in 3-D space. Using stereoscopic displays, Andersen and Kramer (1993; see also Andersen, 1990) showed that flanker interference effects decrease with greater separation between target and distractors not only along the horizontal and vertical dimensions, but also in depth (see also Kooi, Toet, Tripathy, \& Levi, 1994). In the present context, if the target and distractors are perceived on different depth planes in the barriers-around condition, then one would expect smaller interference effects relative to the barriers-below condition, in which all letters might be perceived on the same depth plane because the 3-D barriers are far from the letters and relative size alone might be an insufficient depth cue.

Indeed, the collective results of Experiments 3-5 suggest that multiple depth cues are needed to produce a strong enough impression of depth in the absence of physical barriers. The lack of modulation of flanker interference in Experiments 3 (with 2-D barriers for different-sized targets and distractors) and 5 (with 3-D barriers for equal-sized targets and distractors) suggests that a single depth cue-either 3-D barriers or relative size - is insufficient. The modulation of flanker interference in Experiment 4 (with 3-D barriers for different-sized targets and distractors) suggests that the two depth cues in combination are sufficient. These suggestions are consistent with research showing that more depth is perceived with more cues (Bruno \& Cutting, 1988).

The 3-D referential coding hypothesis represents a novel extension to the referential coding hypothesis of Murchison and Proctor $(2015,2016)$ and an alternative to the hand space hypothesis of Davoli and Brockmole (2012) in accounting for the modulation of flanker interference by physical barrier placement. It is the first account to highlight a possible link between depth perception and attentional allocation in flanker tasks involving barriers. Further research directed toward understanding the generality of referential coding in related situations will likely provide deeper insight regarding contextual effects on visual selective attention.

Author note I thank Allison Blake, Sammi Franklin, Michael Kanczuzewski, Bailey Masterson, Chasity Ricker, and Tessa Stephens for assistance with data collection.

\section{References}

Abrams, R. A., Davoli, C. C., Du, F., Knapp, W. H., \& Paull, D. (2008). Altered vision near the hands. Cognition, 107, 1035-1047.

Andersen, G. J. (1990). Focused attention in three-dimensional space. Perception \& Psychophysics, 47, 112-120.

Andersen, G. J., \& Kramer, A. F. (1993). Limits of focused attention in three-dimensional space. Perception \& Psychophysics, 53, 658667.

Bruno, N., \& Cutting, J. E. (1988). Minimodularity and the perception of layout. Journal of Experimental Psychology: General, 117, 161170.

Bush, W. S., \& Vecera, S. P. (2016). Hand position biases processing toward task irrelevant flankers. Journal of Experimental Psychology: Human Perception and Performance, 42, 151-157.

Davoli, C. C., \& Brockmole, J. R. (2012). The hands shield attention from visual interference. Attention, Perception, \& Psychophysics, 74, 1386-1390.

Eriksen, B. A., \& Eriksen, C. W. (1974). Effects of noise letters upon the identification of a target letter in a nonsearch task. Perception \& Psychophysics, 16, 143-149.

Gratton, G., Coles, M. G. H., Sirevaag, E. J., Eriksen, C. W., \& Donchin, E. (1988). Pre- and poststimulus activation of response channels: A psychophysiological analysis. Journal of Experimental Psychology: Human Perception and Performance, 14, 331-344.

Hommel, B. (1993). The role of attention for the Simon effect. Psychological Research, 55, 208-222.

Kooi, F. L., Toet, A., Tripathy, S. P., \& Levi, D. M. (1994). The effect of similarity and duration on spatial interaction in peripheral vision. Spatial Vision, 8, 255-279.

Miller, J. (1991). The flanker compatibility effect as a function of visual angle, attentional focus, visual transients, and perceptual load: A search for boundary conditions. Perception \& Psychophysics, 49, 270-288.

Murchison, N. M., \& Proctor, R. W. (2015). How hand placement modulates interference from extraneous stimuli. Attention, Perception, \& Psychophysics, 77, 340-352.

Murchison, N. M., \& Proctor, R. W. (2016). Intentional control of visual processing benefits from referential objects. Psychonomic Bulletin \& Review, 23, 1164-1169.

Palmer, S. E. (1999). Vision science: Photons to phenomenology. Cambridge: MIT Press.

Rouder, J. N., Speckman, P. L., Sun, D., Morey, R. D., \& Iverson, G. (2009). Bayesian $t$ tests for accepting and rejecting the null hypothesis. Psychonomic Bulletin \& Review, 16, 225-237.

Tseng, P., Bridgeman, B., \& Juan, C.-H. (2012). Take the matter into your own hands: A brief review of the effect of nearby-hands on visual processing. Vision Research, 72, 74-77.

Weidler, B. J., \& Abrams, R. A. (2014). Enhanced cognitive control near the hands. Psychonomic Bulletin \& Review, 21, 462-469. 